\title{
High-power free-electron laser amplifier using a scalloped electron beam and a two-stage wiggler
}

\author{
D. C. Nguyen \\ Los Alamos National Laboratory, Los Alamos, New Mexico 87545, USA
}

H.P. Freund

Science Applications International Corporation, McLean, Virginia 22102, USA

W. Colson

Naval Postgraduate School, Monterey, California 93943, USA

(Received 16 March 2006; published 8 May 2006)

\begin{abstract}
High-power free-electron laser (FEL) amplifiers present many practical design and construction problems. One such problem is possible damage to any optical beam control elements beyond the wiggler. The ability to increase the optical beam's divergence angle after the wiggler, thereby reducing the intensity on the first optical element, is important to minimize such damage. One proposal to accomplish this optical beam spreading is to pinch the electron beam thereby focusing the radiation as well. In this paper, we analyze an approach that relies on the natural betatron motion to pinch the electron beam near the end of the wiggler. We also consider a step-tapered, two-stage wiggler to enhance the efficiency. The combination of a pinched electron beam and step-taper wiggler leads to additional optical guiding of the optical beam. This novel configuration is studied in simulation using the MEDUSA code. For a representative set of beam and wiggler parameters, we discuss (i) the effect of the scalloped beam on the interaction in the FEL and on the focusing and propagation of the radiation, and (ii) the efficiency enhancement in the two-stage wiggler.
\end{abstract}

DOI: 10.1103/PhysRevSTAB.9.050703

PACS numbers: 41.60.Cr

\section{INTRODUCTION}

High-gain free-electron laser (FEL) amplifiers have been proposed as a viable approach to high-power FEL [1]. Typical high-gain FEL amplifiers employ an electron beam that is "matched" to the wiggler so that the envelope remains constant throughout the wiggler. The main advantage of an amplifier over an oscillator for high-average power FELs is that the amplifier has no resonator optics that can suffer either distortion or damage and, thereby, shut down the FEL interaction. However, since the optical beam produced by the amplifier has low divergence and high power, any optical elements in its path can be damaged if the beam has not expanded to a sufficiently low power density. Consequently, the first optical elements must be placed a long distance from the wiggler to minimize the optical power density on the optical elements. For many high-power FEL designs, however, it is important to shorten that distance by increasing the optical mode's divergence angle (and thus the radius on the first optical elements).

Sprangle et al. [2] first proposed pinching the electron beam with external magnetic focusing elements at the end of the wiggler in an FEL amplifier to increase the divergence of the optical mode beyond the wiggler. Because the optical mode is guided by the interaction with the electron beam, pinching the electron beam will result in pinching of the optical mode. Since the optical mode's divergence angle is inversely proportional to the mode radius, pinching of the electron beam eventually gives rise to more rapid expansion of the optical mode after the wiggler than would be obtained by using an electron beam that is matched to the wiggler.

Using external magnetic focusing elements such as quadrupole focusing magnets introduces variations in the electron beam longitudinal velocity and therefore reduces the strength of the FEL interaction, resulting in decreased optical guiding. In this paper, we present an alternate approach for pinching the electron beam by relying on betatron motion due to natural (weak) focusing to produce a pinched electron beam near the end of the wiggler. The advantage of natural focusing over quadrupole focusing is that the FEL resonance condition of the electrons due to their initial Lorentz factor, transverse position, and transverse angle is not affected by betatron oscillations. The configuration is illustrated schematically in Fig. 1. The electron beam is injected into the wiggler with a radius $\left(\sigma_{f}\right)$ smaller than the matched-beam radius $\left(\sigma_{m}\right)$ so that betatron oscillation places the electron beam waist at one scalloping period $\left(\lambda_{\Sigma}\right)$ from the entrance. The "pinch point" is located within the second wiggler segment of a step-tapered wiggler. Optical guiding in a high-gain FEL typically ends as the interaction saturates and the optical mode starts to expand near the end of the wiggler. However, in this design the second wiggler segment has a smaller $a_{w}$ that maintains the resonant interaction so exponential gain and optical guiding continue to the waist of the scalloped electron beam. The combination of optical guiding and a pinched electron beam causes the optical 


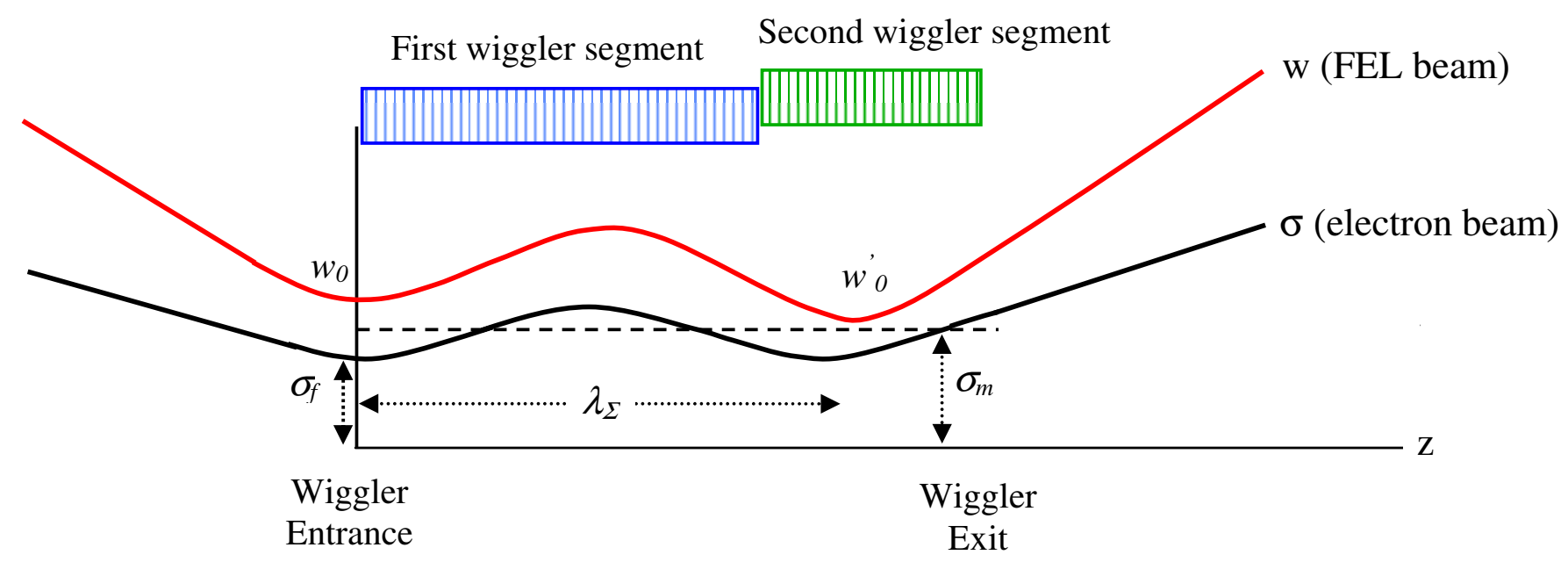

FIG. 1. (Color) Illustration of a scalloped electron beam interaction in a two-stage wiggler.

mode to focus near the exit of the second wiggler, thereby increasing the diffraction angle at the wiggler exit. It is important to recognize that the second wiggler segment performs two functions: (i) to extend, albeit briefly, optical guiding and exponentiation so that focusing of the optical mode continues, and (ii) to enhance the FEL extraction efficiency.

The organization of the paper is as follows. We discuss issues that may arise with using scalloped electron beams in a high-gain FEL in Sec. II. In Sec. III, we describe the three-dimensional simulation code MEDUSA $[3,4]$ that is used to model the FEL interaction with a scalloped electron beam and a step-tapered, two-stage wiggler. Section IV is devoted to discussion of the numerical simulation results. We study, via numerical simulations, the effect of the scalloped beam on the interaction in the FEL and on the focusing and propagation of the radiation, and the efficiency enhancement in the two-stage wiggler.

\section{HIGH-GAIN FEL WITH SCALLOPED ELECTRON BEAMS}

Interest has largely focused on electron beams that are matched to the wiggler in high-gain FEL amplifiers in the sense that the beam envelope maintains a relatively constant size and shape in the wiggler. This configuration puts a stringent requirement on the normalized rms emittance because the matched electron beam radius inside the wiggler depends on the beam emittance. Operating a high-gain FEL with a scalloped electron beam relaxes the beam emittance requirement since the electron beam needs only to come to a waist near the end of the wiggler.

We can write the equation for the evolution of the envelope of a relativistic, axisymmetric beam with equal transverse emittance and equal two-plane focusing as [5]

$$
\frac{d^{2} \sigma}{d z^{2}}=-k_{\beta}^{2} \sigma+\frac{2 I}{\gamma^{3} I_{A} \sigma}+\frac{\varepsilon_{n}^{2}}{\gamma^{2} \sigma^{3}}
$$

with the first term corresponding to betatron focusing, the second term space-charge defocusing and the third term emittance term. The betatron wave number $k_{\beta}$ is

$$
k_{\beta}=\left(\frac{e B_{0}}{2 \gamma m_{e} c}\right)=\frac{k_{w} a_{w}}{\sqrt{2} \gamma}
$$

where $k_{w}=2 \pi / \lambda_{w}$ is the wiggler wave number corresponding to the wiggler period $\lambda_{w} ; a_{w}=e B_{0} / \sqrt{2} k_{w} m_{e} c$ is the wiggler strength parameter corresponding to the peak on-axis field $B_{0} ; \gamma$ is the relativistic factor, $\varepsilon_{n}$ is the normalized rms emittance; $I$ and $I_{A}$ are the beam and Alfvén currents, respectively; $m_{e}$ is the electron rest mass; and $c$ the speed of light in vacuo. The second term of Eq. (1) can be neglected for an emittance-dominated beam. Under the steady-state condition, the emittancedominated matched-beam radius is

$$
\sigma_{0}=\sqrt{\frac{\sqrt{2} \varepsilon_{n}}{k_{w} a_{w}}} .
$$

As the current increases, we can account for its effects by including the space-charge modification to the emittancedominated matched-beam radius

$$
\sigma_{m}=\sigma_{0}\left(u+\sqrt{1+u^{2}}\right)^{1 / 2},
$$

where $u=\sqrt{2}\left(I / I_{A}\right) /\left(\gamma^{2} \varepsilon_{n} k_{\beta}\right)$ is a dimensionless spacecharge parameter [5]. Space charge also modifies the evolution of the envelope for a mismatched beam. Using a first order perturbation analysis of Eq. (1) we can show that

$$
\sigma=\sigma_{f}+\left(\sigma_{m}-\sigma_{f}\right) \sin \left(k_{\Sigma} z\right),
$$

where $k_{\Sigma}$ is defined as the scalloping wave number. In the absence of space charge, $k_{\Sigma}$ is twice the betatron wave number. With space charge, the scalloping wave number is 


$$
k_{\Sigma}=2 k_{\beta} \sqrt{1-\left(\frac{I}{I_{A}}\right) \frac{1}{\gamma^{2} k_{\beta} \varepsilon_{n}}} .
$$

The parameters of interest (see Table I) describe an $80 \mathrm{MeV} / 1000 \mathrm{~A}$ electron beam with an rms normalized emittance of $10 \mathrm{~mm}-\mathrm{mrad}$, and a wiggler field such that the betatron period is about $4.2 \mathrm{~m}$. Using these parameters, the space-charge-induced increase in the matched-beam radius is $10 \%$ and the space-charge-induced increase in the scalloping period is $8 \%$. Hence, space-charge effects on the pinched-beam dynamics are negligible for the case of interest, and need not be included in the simulations of the concept.

The advantages of using a scalloped beam to increase the divergence angle of the optical mode at the wiggler exit can be illustrated by consideration of some simple scaling laws. In the first place, the optical mode in the exponential gain regime is guided by the interaction so that the filling factor is relatively constant and the gain length scales roughly as the one-dimensional theory predicts. Hence, the gain length $L_{G}$ scales with beam radius $\sigma$ roughly as $L_{G} \propto \sigma^{2 / 3}$. The diffraction angle $\theta_{D}$ scales inversely with the spot size $w_{0}$, i.e. $\theta_{D}=\lambda / \pi w_{0}$. Hence, if the optically guided spot size corresponds to the pinched-beam radius $\left(w_{0} \approx \sigma\right)$, then the diffraction angle will scale roughly as $\theta_{D} \propto \sigma^{-1}$. As a result, the effect of the pinched beam on the gain length will be relatively weak, but it will have a significant effect on increasing the diffraction angle.

Saturation occurs in an FEL when the bulk of the electrons are trapped in the ponderomotive potential and exe-

TABLE I. FEL and beam parameters used in MEDUSA simulations.

\begin{tabular}{lll}
\hline \hline \multicolumn{1}{c}{ FEL Parameters } & Symbols & Values \\
\hline Beam energy & $E_{b}$ & $80.8 \mathrm{MeV}$ \\
Peak current & $I$ & $1 \mathrm{kA}$ \\
rms Emittance (normalized) & $\varepsilon_{n}$ & $10 \mathrm{~mm}-\mathrm{mrad}$ \\
rms Energy spread & $\Delta \gamma / \gamma$ & $0.25 \%$ \\
Wiggler period & $\lambda_{w}$ & $2.18 \mathrm{~cm}$ \\
1st Wiggler section field & $B_{w l}$ & $8.247 \mathrm{kG}$ \\
1st Wiggler section parameter & $a_{w l}$ & 1.187 \\
1st Wiggler section length & $L_{w l}$ & $1.918 \mathrm{~m}$ \\
2nd Wiggler section field & $B_{w 2}$ & $8.032 \mathrm{kG}$ \\
2nd Wiggler section parameter & $a_{w 2}$ & 1.156 \\
2nd Wiggler section length & $L_{w 2}$ & $0.480 \mathrm{~m}$ \\
Matched-beam radius & $\sigma_{m}$ & $0.23 \mathrm{~mm}$ \\
Beam radius at wiggler entrance & $\sigma_{f}$ & $0.11 \mathrm{~mm}$ \\
Injected radiation peak power & $P_{\text {in }}$ & $1 \mathrm{MW}$ \\
Injected radiation waist & $w_{0}$ & $0.305 \mathrm{~mm}$ \\
Output radiation peak power & $P_{\text {out }}$ & $1.45 \mathrm{GW}$ \\
FEL extraction efficiency & $\eta_{1}$ & $1.8 \%$ \\
Output radiation minimum waist & $w_{0}$ & $0.258 \mathrm{~mm}$ \\
Output radiation Rayleigh range & $z_{R}$ & $6.36 \mathrm{~cm}$ \\
\hline \hline
\end{tabular}

cute half of a synchrotron oscillation so that as many electrons are losing energy to the wave as gaining energy from it. It is well known that a linear-tapered wiggler can prolong the interaction and increase the extraction efficiency. Improved FEL performance can also be achieved using a step-tapered wiggler [6]. In either case, the taper must begin after the electrons become trapped in the ponderomotive potential but before saturation is reached. However, optical guiding is weak in the FEL interaction in a linear-tapered wiggler. In contrast, exponential growth can be maintained in the second wiggler segment in a steptapered wiggler configuration, which also results in further optical guiding. Moreover, the energy spread of the electron beam exiting the step-tapered wiggler is smaller than in the linear-tapered wiggler.

A MATHCAD program using the PERSEO library has been used to illustrate the FEL interaction in the steptapered wiggler [7]. As shown in the example in Fig. 2, the phase-space distributions at the end of the first wiggler segment (a) and the second wiggler segment (b) show the electrons undergo additional deceleration in the second wiggler segment. The resonant energy, $\gamma_{1}=\left[\lambda_{w}(1+\right.$ $\left.\left.a_{w}^{2}\right) / 2 \lambda\right]^{1 / 2}$, of the first wiggler segment is 158.1 (red, dashed line). At the end of the first wiggler segment, the electrons have rotated to below the resonance energy $\left(\gamma_{1}\right)$.
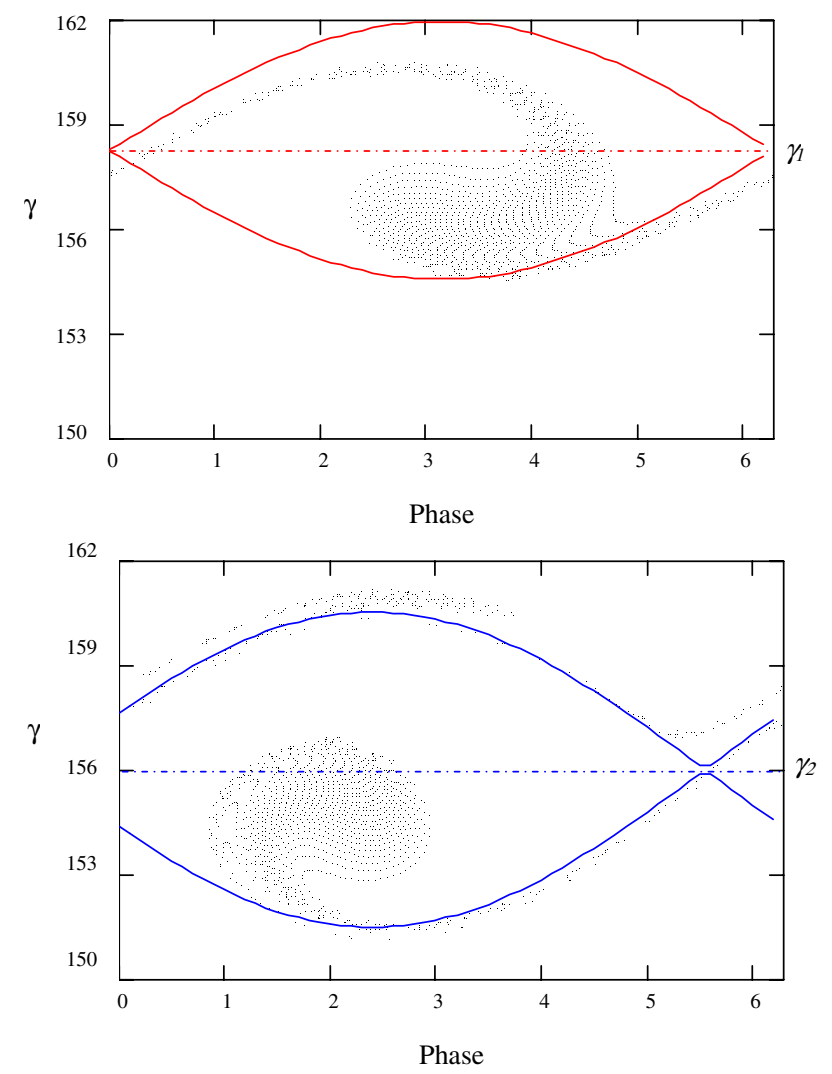

(b)

FIG. 2. (Color) Longitudinal phase space (a) at the end of the first wiggler segment, and (b) at the end of the second wiggler segment. 
The electron beam average $\gamma$ has been reduced from the injected value of 159.1 to 157.0 , or $1.3 \%$ extraction efficiency. Efficiency can be enhanced in a two-stage wiggler where the second wiggler has lower $a_{w}$ (or shorter wiggler period) and thus a lower resonant $\gamma_{2}$. In the example, the second wiggler segment has a resonant $\gamma_{2}$ of 155.8 (blue, dashed line). Consequently, the electrons continue to rotate in the second wiggler's ponderomotive potential to a lower average $\gamma$ of 155.3 , or $2.4 \%$ extraction efficiency.

\section{THE NUMERICAL FORMULATION}

This configuration is studied in simulation using the MEDUSA code [3,4], which employs a three-dimensional formulation that includes the slippage of the radiation relative to the electron beam. MEDUSA can model both helical and planar wiggler geometry and treats the electromagnetic field as a superposition of either Gauss-Hermite or Gauss-Laguerre modes in the slowly varying amplitude approximation. We use the Gauss-Hermite modes in conjunction with a two-plane focusing wiggler in this work; hence,

$\delta \mathbf{A}(\mathbf{x}, t)=\hat{\mathbf{e}}_{x} \sum_{l, n, h} e_{l, n, h}(x, y)\left[\delta A_{l, n, h}^{(1)} \cos \varphi_{h}+\delta A_{l, n, h}^{(2)} \sin \varphi_{h}\right]$,

where $l$ and $n$ are transverse mode numbers, $h$ is the harmonic number, $e_{l, n, h}=\exp \left(-r^{2} / w_{h}^{2}\right) H_{l}\left(\sqrt{2} x / w_{h}\right) \times$ $H_{n}\left(\sqrt{2} y / w_{h}\right), H_{l}$ is the Hermite polynomial of order $l$, and $w_{h}$ is the spot size, $\varphi_{h}=h\left(k_{0} z-\omega_{0} t\right)+$ $\alpha_{h} r^{2} / w_{h}{ }^{2}\left(k_{0}=\omega_{0} / c\right)$. We assume that $\delta A_{l, n, h}^{(1,2)}, w_{h}$, and $\alpha_{h}$ vary slowly in $z$ and $t$. The dynamical equations are

$$
\left(\frac{d}{d z}+\frac{w_{h}{ }^{\prime}}{w_{h}}\right)\left(\begin{array}{c}
\delta a_{l, n, h}^{(1)} \\
\delta a_{l, n, h}^{(2)}
\end{array}\right)+K_{l, n, h}\left(\begin{array}{c}
\delta a_{l, n, h}^{(2)} \\
-\delta a_{l, n, h}^{(1)}
\end{array}\right)=\left(\begin{array}{c}
s_{l, n, h}^{(1)} \\
s_{l, n, h}^{(2)}
\end{array}\right)
$$

where $\delta a_{l, n, h}^{(1,2)}=e \delta A_{l, n, h}^{(1,2)} / m_{e} c^{2}, d / d z=\partial / \partial z+c^{-1} \partial / \partial t$, the "prime" superscript denotes the total $z$-derivative,

$$
\begin{gathered}
K_{l, n, h}=(l+n+1)\left(\alpha_{h} \frac{w_{h}^{\prime}}{w_{h}}-\frac{\alpha_{h}^{\prime}}{2}-\frac{1+\alpha_{h}^{2}}{h k_{0} w_{h}^{2}}\right), \\
\left(\begin{array}{c}
s_{l, n, h}^{(1)} \\
s_{l, n, h}^{(2)}
\end{array}\right)=\frac{2 \omega_{b}^{2}}{h \omega_{0} c} \frac{F_{l, n}}{w_{h}^{2}}\left\langle\frac{v_{x}}{\left|v_{z}\right|} e_{l, n, h}\left(\begin{array}{c}
\cos \varphi_{h} \\
-\sin \varphi_{h}
\end{array}\right)\right\rangle,
\end{gathered}
$$

where $\omega_{b}(z, t)^{2}=4 \pi e^{2} n_{b}(z, t) / m_{e}$ for a beam density $n_{b}$, and $F_{l, n}=\left[2^{l+n} l ! n !\right]^{-1}$. The spot size and radius of curvature for each harmonic component are given by

$$
\begin{gathered}
w_{h}^{\prime}=\frac{2 \alpha_{h}}{h k_{0} w_{h}}-w_{h} Y_{h}, \\
\frac{\alpha_{h}^{\prime}}{2}=\frac{1+\alpha_{h}^{2}}{h k_{0} w_{h}^{2}}-\left(X_{h}+\alpha_{h} Y_{h}\right) .
\end{gathered}
$$

These equations constitute the source-dependent expansion [8], which is a self-consistent adaptive eigenmode representation that tracks the optical guiding of the mode based upon the interaction with the electron beam. The field equations are integrated simultaneously with the complete three-dimensional Lorentz force equations for an ensemble of electrons. No wiggler-average orbit approximation is used so that the spatial step size must be small enough to resolve the wiggler motion.

\section{SIMULATION RESULTS AND DISCUSSION}

Table I summarizes the FEL and beam parameters used in the MEDUSA simulations. These parameters are chosen for a 1.05-micron wavelength where high-power seed lasers exist. The electron parameters are challenging but achievable via a high-brightness radio-frequency injector in combination with a magnetic chicane buncher [9]. The wiggler is a conventional permanent-magnet design with parabolic pole faces to provide equal two-plane, sextupole focusing [10]. The wiggler has two segments with slightly different rms wiggler parameters $\left(a_{w}\right)$. We chose an input peak power of $10^{6} \mathrm{~W}$ (for instance, $1 \mu \mathrm{J}$ pulse energy and 1 ps FWHM) to achieve saturation in the $1.9-\mathrm{m}$ first wiggler segment. The scalloping period of $2.1 \mathrm{~m}$ puts the

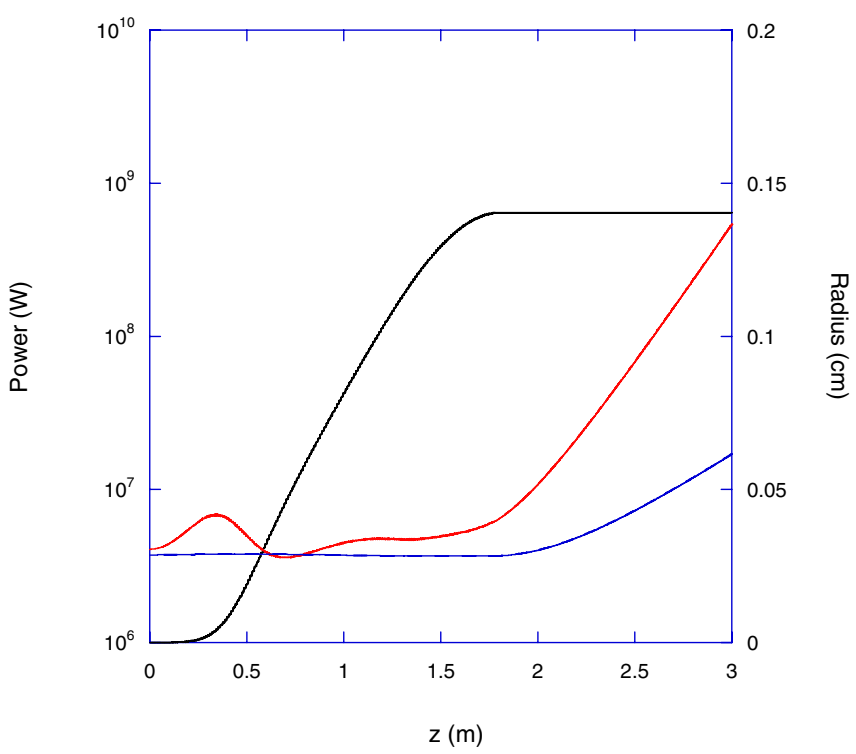

FIG. 3. (Color) Power growth (black), optical beam radius (red), and electron beam radius (blue) inside a matched-beam FEL with a uniform wiggler. 


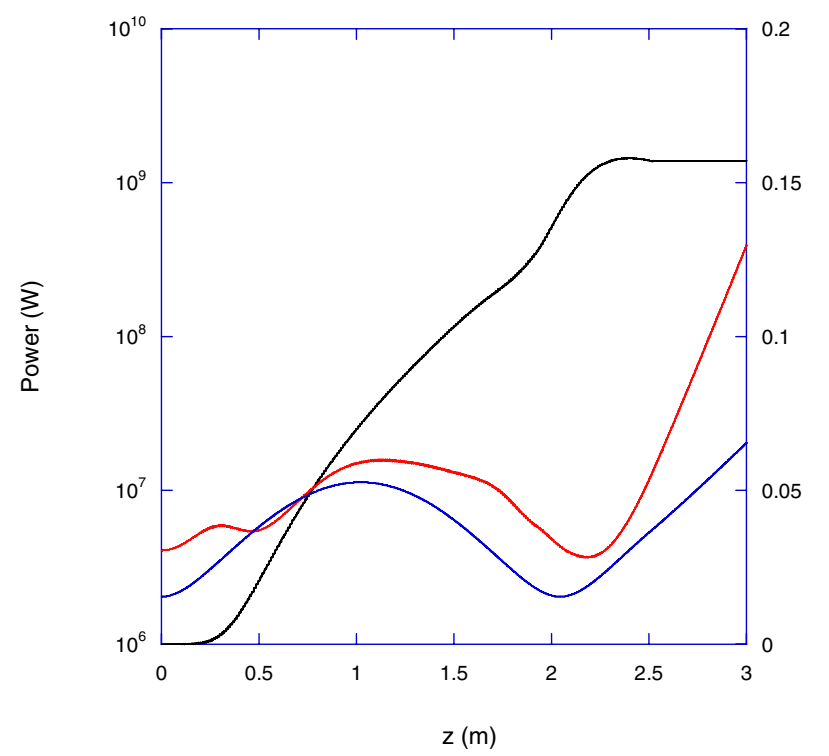

FIG. 4. (Color) Power growth (black), optical beam radius (red), and electron beam radius (blue) inside a scalloped-beam FEL with a two-stage wiggler.

second waist of the electron beam envelope in the exponential gain region of the $0.48-\mathrm{m}$ second wiggler segment.

We first consider the case of a uniform wiggler with the same $a_{w}$ as the first segment but the length is $1.9 \mathrm{~m}$. Figure 3 shows the optical power (black), optical beam radius (red), and electron beam radius (blue) in this wiggler. The optical mode is guided up to a point just shy of saturation (at $1.75 \mathrm{~m}$ ). As saturation is approached, optical guiding is reduced and the optical beam radius increases near the end of the wiggler.

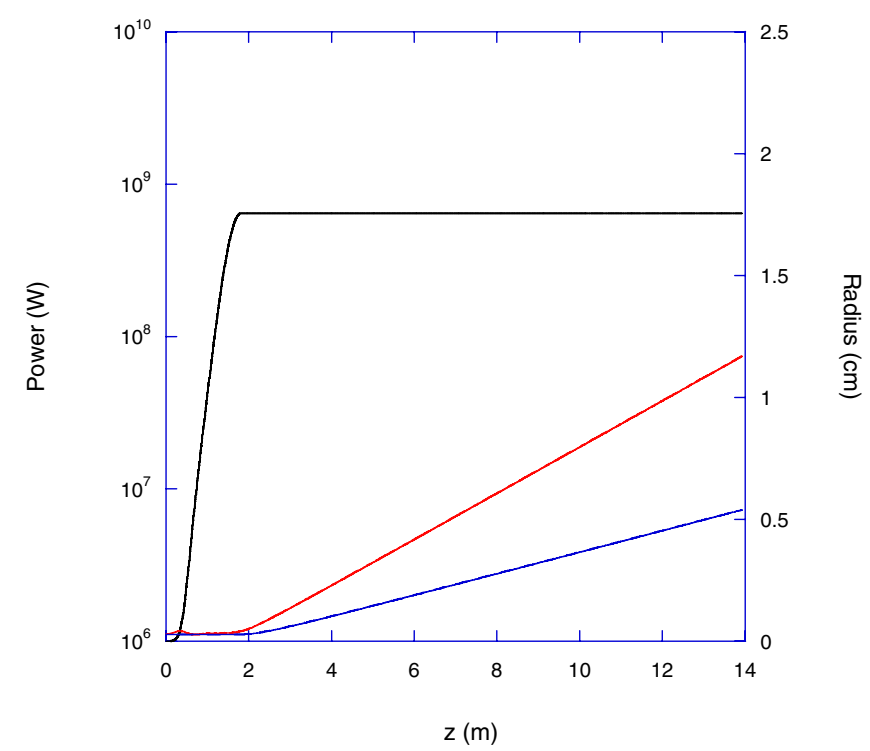

FIG. 5. (Color) Power (black), optical beam radius (red), and electron beam radius (blue) over a large distance for a matchedbeam FEL with a uniform wiggler.

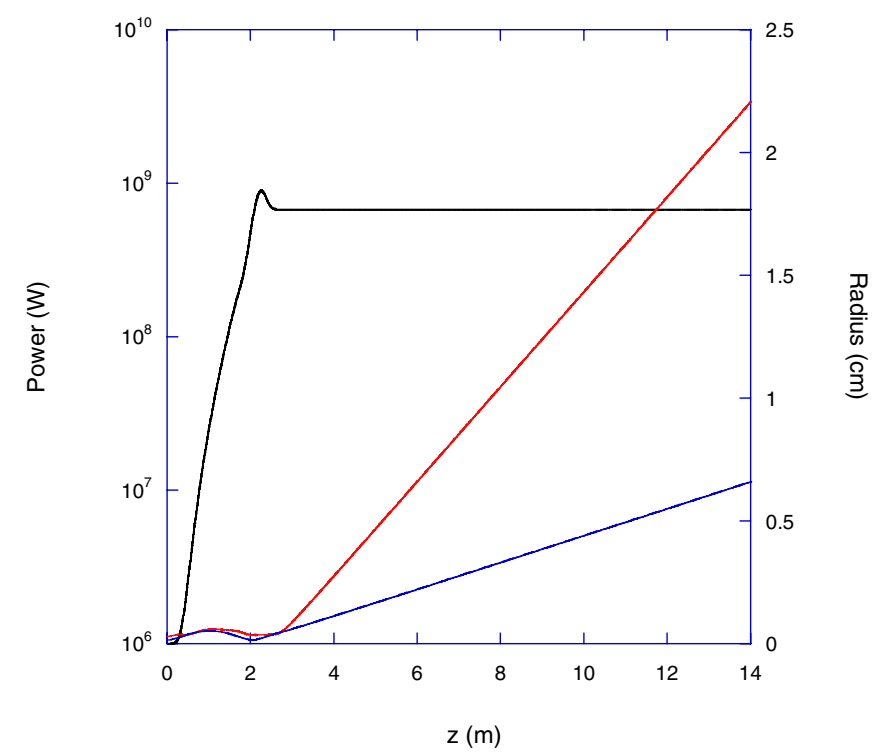

FIG. 6. (Color) Power (black), optical beam radius (red), and electron beam radius (blue) (green) over a large distance for a scalloped-beam FEL with a uniform wiggler.

We now consider the case of a scalloped electron beam with the two-stage wiggler shown in Table I. The optical power (black), optical beam radius (red), and electron beam radius (blue) are shown in Fig. 4. The second minimum of the optical beam radius at $z=2.15 \mathrm{~m}$ illustrates the reestablishment of optical guiding as the electron beam comes to a second waist (at $z=2.05 \mathrm{~m}$ ) in the second wiggler segment where exponential gain continues $(z=$ $2-2.5 \mathrm{~m})$.

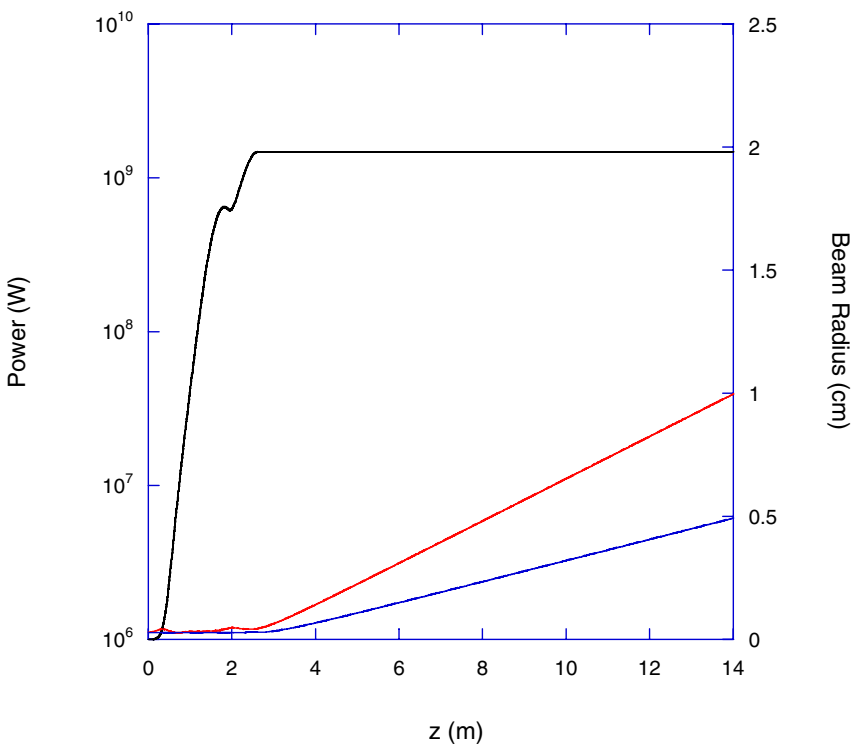

FIG. 7. (Color) Power (black), optical beam radius (red), and electron beam radius (blue) over a large distance for a matchedbeam FEL with a two-stage wiggler. 


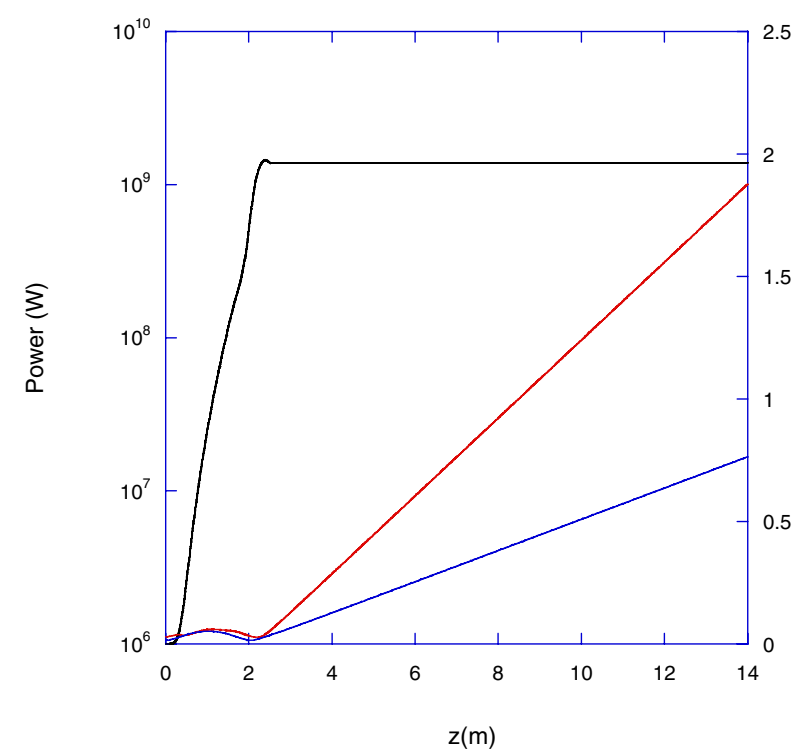

FIG. 8. (Color) Power (black), optical beam radius (red), and electron beam radius (blue) over a large distance for a scallopedbeam FEL with a two-stage wiggler.

The two cases shown in Figs. 3 and 4 have implications for the divergence of the optical mode beyond the wiggler exit. The propagation of the optical mode beyond the wiggler exit is shown in Figs. 5 and 6 for a uniform wiggler and matched and scalloped electron beams, respectively. Again we plot the optical power (black), optical beam radius (red), and electron beam radius (blue) but over a distance up to $14 \mathrm{~m}$. For the case of matched beam in a uniform wiggler (Fig. 5), the power reaches a maximum of $650 \mathrm{MW}$ at $z=1.9 \mathrm{~m}$, and the optical beam expands to $11.2 \mathrm{~mm}$ at $z=14 \mathrm{~m}$ yielding a divergence half-angle of $0.9 \mathrm{mrad}$. For the case of a scalloped beam with a uniform wiggler (Fig. 6), the power reaches a maximum of $0.78 \mathrm{GW}$ at $z=2.14 \mathrm{~m}$, and the optical beam expands to $22.1 \mathrm{~mm}$ at $z=14 \mathrm{~m}$ yielding a divergence half-angle of $2.2 \mathrm{mrad}$. This is more than a factor of 2 increase in the divergence angle over the matched-beam, uniform wiggler case, and translates into a factor-of-five reduction in the optical intensity on the first optical element.

The effects of the two-stage wiggler are illustrated in Figs. 7 and 8, which correspond to Figs. 5 and 6, except the uniform wiggler is replaced with a two-stage wiggler. Figures 7 and 8 show the optical power (black), optical beam radius (red), and electron beam radius (blue) for a matched beam (Fig. 7) and a scalloped beam (Fig. 8). In the matched-beam, two-stage wiggler case (Fig. 7), the power reaches a maximum of $1.48 \mathrm{GW}$ at $z=2.6 \mathrm{~m}$ and optical guiding is partially reestablished in the second wiggler segment. Compared to the case of matched beam in a uniform wiggler (Fig. 5), the divergence half-angle decreases slightly (from 0.9 to $0.86 \mathrm{mrad}$ ). In the scallopedbeam, two-stage wiggler case (Fig. 8), the power reaches a maximum of $1.45 \mathrm{GW}$, almost the same as the matched-

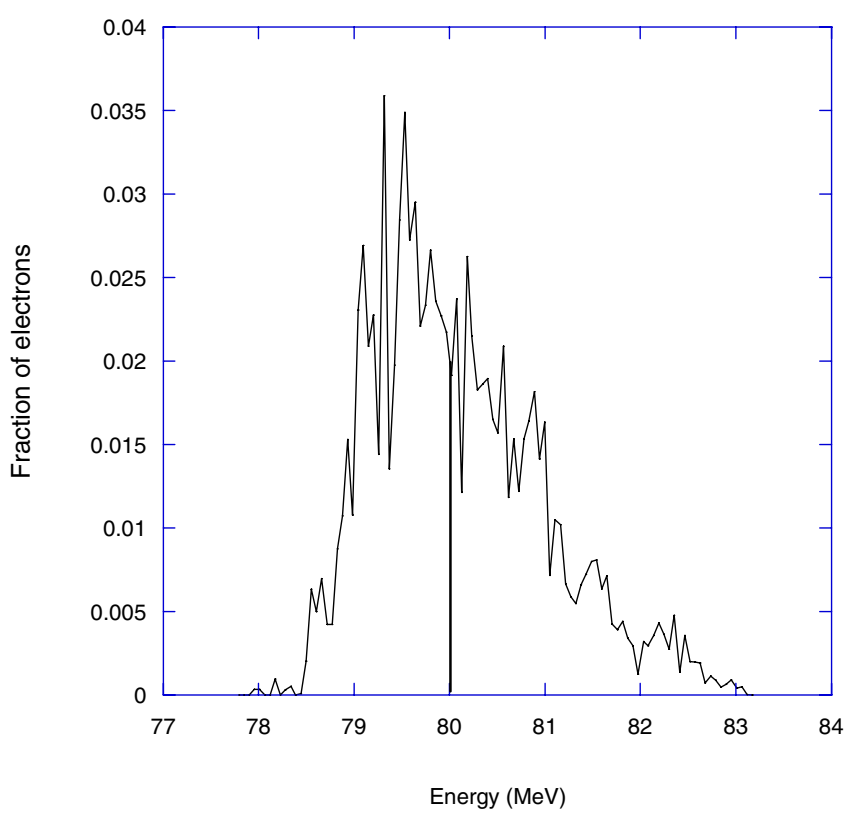

(a)

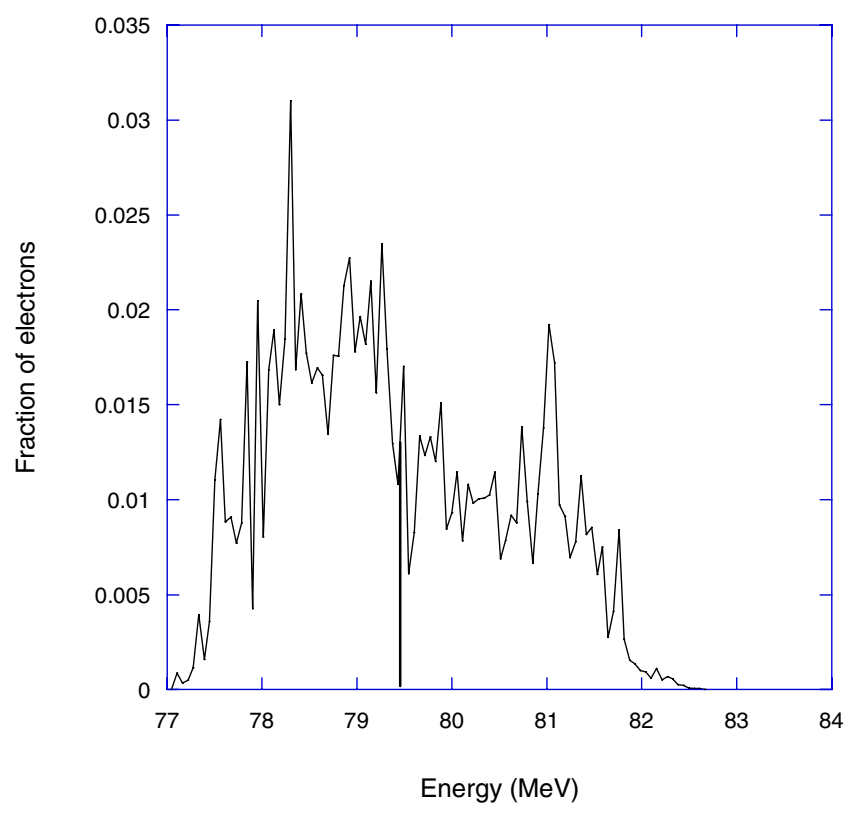

(b)

FIG. 9. (Color) Spent beam distribution from (a) the uniform wiggler, and (b) the two-stage wiggler.

beam case, but the divergence half-angle is $1.6 \mathrm{mrad}$, almost twice the matched-beam, two-stage wiggler case $(0.86 \mathrm{mrad})$, and 1.7 times the matched-beam, uniform wiggler case $(0.9 \mathrm{mrad})$.

The average energy and energy distribution of the spent electron beams for the uniform wiggler and two-stage wiggler are shown in Figs. 9(a) and 9(b), respectively. The average electron beam energy at the end of the uniform wiggler is $80.0 \mathrm{MeV}$, corresponding to $1.0 \%$ extraction. The average electron beam energy at the end of the 


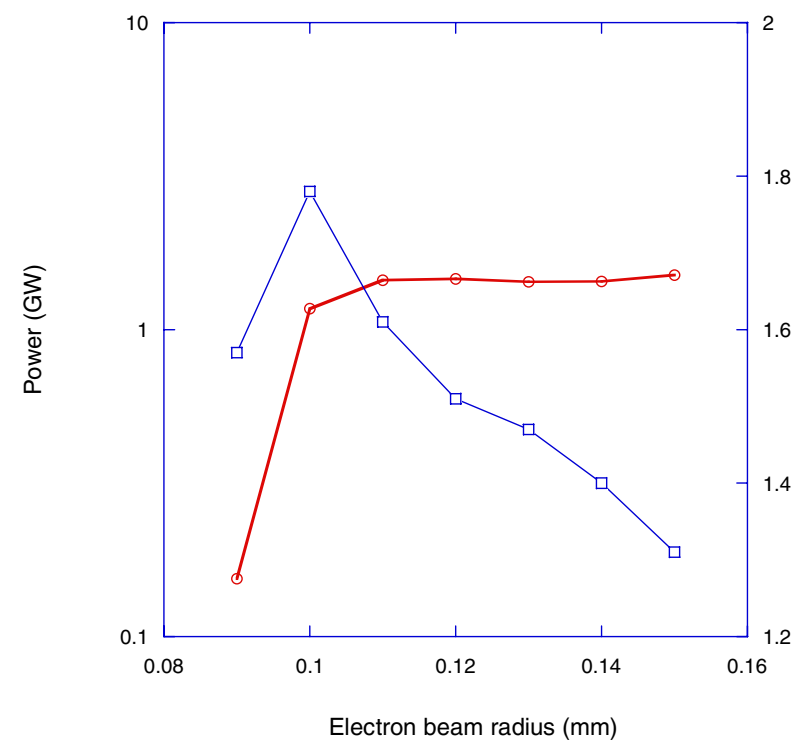

FIG. 10. (Color) Dependence of optical power (circle) and divergence half-angle (square) on the minimum electron beam radius.

two-stage wiggler is $79.35 \mathrm{MeV}$, corresponding to $1.8 \%$ extraction. The full energy spread (base width) increases from $5.32 \mathrm{MeV}(6.7 \%)$ for the uniform wiggler to $5.62 \mathrm{MeV}(7.1 \%)$ for the two-stage wiggler. Thus, using the two-stage wiggler increases the efficiency by a factor of 1.8. The penalties with using the two-stage wiggler are a $25 \%$ reduction in the divergence angle (from 2.2 to $1.6 \mathrm{mrad}$ ) and a slight increase in the energy spread of the spent electron beam (from $6.7 \%$ to $7.1 \%$ ).

We now consider the effect of focusing the electron beam to different radii at the wiggler entrance, keeping the beam emittance constant. As shown in Fig. 10, reducing the rms radius of the electron beam at first increases the FEL divergence angle without affecting the peak power. However, below the beam radius of $0.1 \mathrm{~mm}$, there is a significant reduction in the peak power and a concomitant reduction in the FEL divergence angle. The optimum electron beam radius is approximately $0.11 \mathrm{~mm}$ where both the divergence angle and peak power remain large.

We also optimize the lengths of the first and second wiggler segments for maximum efficiency enhancement (Fig. 11). The optimum length for the two wiggler segments is about $2.4 \mathrm{~m}$ (110 periods). This is $7 \%$ longer than the saturation length of the uniform wiggler with a scalloped electron beam (103 periods). The maximum enhancement factor of 1.9 is obtained with 88 periods in the first segment, corresponding to the optimum prebunching of the electrons prior to injection into the second segment.

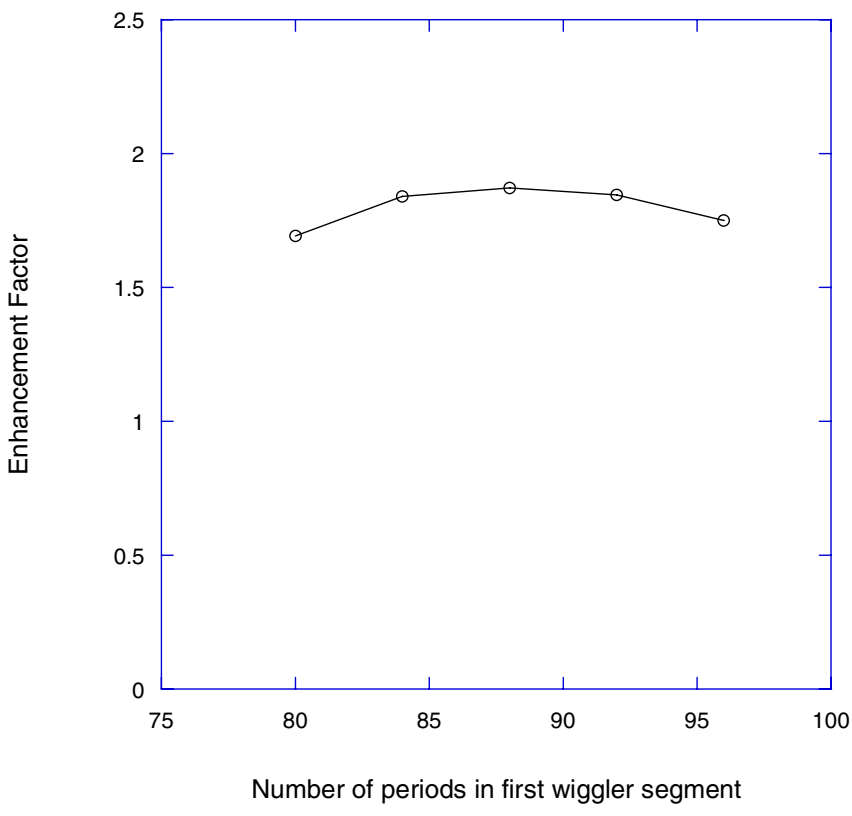

FIG. 11. (Color) Efficiency enhancement of the two-stage wiggler versus the number of periods in the first wiggler segment.

\section{ACKNOWLEDGMENTS}

We thank the Office of Naval Research for financial support. We are also grateful to Bruce Carlsten for helpful discussion and Luca Gianessi for the MATHCAD program to calculate the electron longitudinal phase-space distribution in a two-stage wiggler.

[1] D. C. Nguyen and H. P. Freund, Nucl. Instrum. Methods Phys. Res., Sect. A 507, 120 (2003).

[2] P. Sprangle, B. Hafizi, and J. R. Peñano, IEEE J. Quantum Electron. 40, 1739 (2004).

[3] H. P. Freund, S. G. Biedron, and S. V. Milton, IEEE J. Quantum Electron. 36, 275 (2000).

[4] H. P. Freund, Phys. Rev. ST Accel. Beams 8, 110701 (2005).

[5] M. Reiser, Theory and Design of Charged Particle Beams (Wiley, New York, 1994).

[6] J. Blau et al., Nucl. Instrum. Methods Phys. Res., Sect. A 483, 138 (2002).

[7] L. Giannessi, PERSEO reference available at www. perseo.enea.it.

[8] P. A. Sprangle, A. Ting, and C. M. Tang, Phys. Rev. A 36, 2773 (1987).

[9] B. Carlsten and S. Russell, Phys. Rev. E 53, R2072 (1996).

[10] E. T. Scharlemann, J. Appl. Phys. 58, 2154 (1985). 